\title{
When does an EEG contribute to the management of febrile seizures?
}

\author{
Gregory Stores
}

The above question implies that the electroencephalogram (EEG) can be of some value in the care of children with febrile seizures. Not everyone would agree. For example Aicardi states uncompromisingly that 'EEG tracings are useless as they do not help predict the occurrence of later epilepsy'. ${ }^{1}$ Some accounts are so disdainful that they do not mention electroencephalography at all. ${ }^{2}$ However other authors are less sure: 'The role of the electroencephalogram in the work-up of febrile seizures remains controversial'. 3

The possible value of the EEG in these circumstances depends to some extent on the definition of febrile seizures and the possible identification of different types. Both are still contentious issues.

Regarding definition, the National Institutes of Health (NIH) consensus statement excluded seizures with evidence of intracranial infection or a definite cause, and also children with previous non-febrile seizures. ${ }^{3}$ Although these criteria are accepted in some child neurology texts (for example, Menkes ${ }^{4}$ ), other authors point to the difficulties of achieving a strict definition in these terms, for example Brett. ${ }^{5}$ Aicardi in particular is critical of the NIH consensus definition: 'The absence of intracranial infection and of recognised acute neurological illness remains an unproved assumption in at least some cases'.

As for subgroups, there is general (though not complete) agreement with the distinction made by Livingstone between 'simple febrile convulsions' and 'epileptic seizures precipitated by fever' or 'atypical febrile convulsions' which are also sometimes called 'complex' or 'complicated' febrile seizures. ${ }^{6}$ In Livingstone's account simple febrile seizures, accounting for $\mathbf{9 7 \%}$ of the total, were brief generalised seizures in children with a family history of febrile seizures, in contrast to atypical febrile seizures that were usually prolonged and often focal or lateralised. Only 3\% of the former group developed later afebrile seizures compared with $93 \%$ of the latter group. In more recent years it has been claimed that this poor prognosis group is further characterised by such features as neurological or developmental abnormality before the onset of seizures, onset under the age of 1 year, and frequent or prolonged febrile seizures.

These descriptions begin to suggest a possible role for electroencephalography in helping to identify the nature of underlying cerebral pathology in children assigned to the atypical group. However Livingstone's neat distinction between simple and atypical febrile seizures has also been challenged, again perhaps particularly by Aicardi who asserts that although a large majority of simple febrile seizures are brief, bilateral and benign in outcome, some children have a combination of simple febrile seizures and long lasting unilateral seizures in spite of a strong family history of febrile seizures. ${ }^{1}$ Chevrie and Aicardi point out that even some febrile seizures with residual neurological signs are not necessarily followed by epilepsy. ${ }^{7}$ In addition the severity of the attacks may not be a good predictor of outcome as almost $90 \%$ of patients who develop epilepsy have had only brief bilateral seizures. ${ }^{8}$ In a later publication Aicardi and Chevrie concluded that unilateral, prolonged febrile seizures are a heterogeneous group containing some children who have seizures because of genetic vulnerability to fever but also others who convulse because of acquired brain injury. 9 Aicardi concludes: 'simple seizures-defined as brief, bilateral attacks in previously normal children-are not necessarily true febrile convulsions, in the sense of seizures entirely resulting from an abnormal constitutional response to fever. ${ }^{1}$ Conversely, complex febrile convulsions or febrile statuses can be true febrile convulsions, . . . the complete separation between simple and atypical febrile convulsions reported by Livingstone has not been confirmed in any other series'. This view should not be construed as excluding the possibility of defining in the future convincing subgroups of children with febrile seizures. Indeed, for example, Aicardi himself points out that febrile seizures of early onset and long duration with only a low grade fever and a positive response to photic stimulation on electroencephalography can be the first manifestation of 'severe myoclonic epilepsy of infants' ${ }^{10}$

Reports of EEG abnormalities in children with febrile seizures (table)

(1) Ictal EEGs

Recordings during acute febrile illness with seizures have rarely been reported. They can be expected to show generalised rapid spiking as in any tonic or tonic-clonic seizure but according to Lennox-Buchtal some show severe lateralised electroencephalographic changes of this type. ${ }^{11}$ In her study, however, no distinction was made between simple and atypical febrile seizures. 
EEG abnormalities in children with a history of febrile seizures

\begin{tabular}{ll}
\hline Ictal (rarely reported) & $\begin{array}{l}\text { Generalised spiking } \\
\text { Lateralised spike wave discharge } \\
\text { Postictal }\end{array}$ \\
Slow activity \\
Spike wave or spikes \\
Bisynchronous theta activity \\
Bisynchronous spike wave at rest \\
and during overbreathing \\
Bisynchronous spike wave on photic \\
stimulation \\
Focal spikes or sharp waves \\
Hypnagogic paroxysmal spike wave
\end{tabular}

\section{(2) Postictal EEGs}

(a) Recordings taken on the same day as the seizure have been reported to contain slow activity in as many of $88 \%$ of cases. The same type of abnormality is described in about a third of children between three and seven days after a seizure. ${ }^{12}$ This slow activity is predominantly posterior in distribution and, although mostly bilateral, is often asymmetrical or even unilateral. It tends to resolve over about a week. The reports by Pampiglione ${ }^{13}$ and Wallace and Zealley ${ }^{14}$ suggested that such abnormalities can be expected in association with viral illnesses whether or not seizures occur, although not in relation to changes of temperature. ${ }^{15}$ However Rantala et al found no difference in the early electroencephalographic findings (including the presence of abnormal slow activity as well as spikes or sharp waves) between those with and those without a proved viral infection. ${ }^{16}$

(b) Spikes or spike and wave discharges, localised or generalised, are rare in the early postictal period. Frantzen et al gave a figure of $1 \cdot 4 \%^{12}$ and Kajitani et al suggest 3\%. ${ }^{17}$ Both these figures are close to the $1.9 \%$ given by EegOlofsson ${ }^{18}$ as the incidence of focal sharp activity in normal young children.

Rantala et al suggest that the presence of any of these abnormalities does not distinguish between simple febrile seizures (defined in their study as lasting less than 15 minutes and without focal features) and complicated febrile seizures (that is, lasting longer than 15 minutes, with focal features or recurrence within 24 hours). ${ }^{16}$ In this investigation febrile seizures were defined as a first seizure associated with fever irrespective of age and excluding children with earlier non-febrile seizures, evidence of a brain infection or metabolic encephalopathy.

\section{(3) Serial EEGs over several years}

Frantzen et al reported that $29 \%$ of children showed some form of 'epileptic' activity in the few years after their last febrile seizure. ${ }^{12}$ Doose et al carried out a longer electroencephalographic follow up study on children with febrile seizures to the age of 11 to 13 years and reported that $81 \%$ had abnormal EEG patterns. ${ }^{19}$

These authors described three types of abnormality all considered to represent genetically determined patterns: (a) bilaterally synchronous and regular 4-7 cycles per second (theta) rhythms seen mainly in parietal regions occurring in $54 \%$ of his series of children with febrile seizures. (b) Generalised spike wave discharge occurring in the resting EEG or in response to overbreathing. This pattern was seen in $49 \%$ with age of onset usually between 3 and 6 years. (c) Generalised spike wave in response to photic stimulation was seen in $42 \%$ mainly in children under the age of 5 years.

From this and other reports it appears that generalised spike wave discharge is unusual at the younger age for febrile seizures (that is, under 2 years) appearing on average about sixteen months after the first febrile seizure up to as late as four years after it. ${ }^{11}{ }^{20}$ The discharges can be intermittent with sometimes months or years apparently in abeyance, although the sampling error of repeated brief electroencephalographic recordings has to be remembered.

Focal spike or sharp wave discharges have been reported in between $8 \%$ and $10 \%$ especially where a persistent slow have focus was seen previously usually after a prolonged seizure. ${ }^{1}$ However, such discharges are also seen after brief febrile convulsions, looking very much like centrotemporal spikes of later childhood. ${ }^{21}$ Kajitani et al reported this finding in $4 \cdot 2 \%$ of children on follow up. ${ }^{17}$

Another obvious abnormality in the later EEGs of children with febrile seizures is that described by Alvarez et al. ${ }^{22}$ They emphasised the occurrence of generalised, paroxysmal, high amplitude rhythmic 3-5 cycles per second slow activity with intermixed spikes in drowsiness ('hypnagogic paroxysmal spike wave activity' or 'hypnagogic PSW') in perhaps a quarter of children with febrile seizures, mainly in the second year of life and rarely younger than this. This feature was not seen in a group of normal controls and appeared in only $9 \%$ of a group of children referred for electroencephalography for neurological problems but without febrile seizures. There appeared to be no clinical difference between children in the febrile seizure group with or without the EEG abnormality.

\section{Prospective studies}

These various abnormalities are very interesting but there seems to be universal agreement that none of them bears any convincing association with the eventual development of epilepsy (that is, recurrent afebrile seizures of primarily cerebral origin) in these children. Some key studies of febrile convulsions concerned with risk factors for later afebrile seizures did not specifically assess the role of electroencephalography ${ }^{23}{ }^{24}$ However the discussion in the NIH consensus statement on febrile seizures is instructive on this point. ${ }^{25}$ The discussion started with the assertion that on general principles electroencephalography findings ought to be predictive of later epilepsy and therefore should form one of the considerations on which each child's management should be based. However, a series of studies were than quoted by Hauser (USA), Thorn (Denmark), and Camfield (Canada) all of which indicated no association between early electroencephalography findings and later seizures. Incidentally, these general conclusions seem to apply to boys and girls equally. 
Conclusions concerning the clinical value of EEG investigations in children with febrile seizures

Clinical value

An early postictal standard EEG will not be helpful in the following respects: (a) it will not distinguish between clinically simple and atypical seizures and (b) it will not particularly help in the identification of a cerebral infective aetiology. (c) EEG findings lack predictive value for the later occurrence of either further febrile or later afebrile seizures. As Aicardi says, epileptic activity will be found in a high proportion of older children who are less likely to develop recurrent seizures and later epilepsy, whereas they will almost never appear in the EEGs of young infants that are at maximum risk of recurrence, later afebrile seizures, and severe febrile seizures. ${ }^{1}$ It is these discrepancies that lead Camfield to say in the above NIH discussion that 'It makes more trouble than it is worth' to carry out recordings in these circumstances; 'you wish you had not done the EEG', because with an abnormal result discussions with parents about its significance can be very difficult. For these reasons electroencephalography cannot be recommended as a routine measure in children with a febrile seizure.

Perhaps, however, there is a limited place for electroencephalographic studies in connection with febrile seizures in those cases where there is clinical reason to suspect cerebral pathology. If the child showed developmental delay or other evidence of neurological impairment before the occurrence of the febrile seizures, or if the first seizure occurs below the age of 12 months, or if the seizures contain partial (focal) features in the ictal or postictal phase, it seems prudent to consider the possibility that the child's seizures may be evidence of brain pathology, perhaps structural. From the above account it is clear that these clinical features do not necessarily suggest a brain lesion and in most cases the findings on further investigation will be normal, but in some instances they will be found to indicate a significant brain abnormality.

In addition, if a child has suffered a prolonged febrile seizure, especially if it is followed by residual neurological signs or developmental regression, the possibility of structural change caused by the convulsions needs to be considered and investigated in a similar way in view of the evidence that various degrees of brain damage can be sustained during the course of a prolonged febrile seizure. ${ }^{9}$

Electroencephalography is not the investigation of choice in considering structural pathology and computed tomography or magnetic resonance imaging would be more appropriate. It can be a useful ancillary investigation capable of suggesting persistent brain pathology (even in the presence of a normal computed tomogram) if it demonstrates an abnormality (usually irregular slow wave disturbance with or without associated spike or sharp wave discharges) which is persistent. Clearly this implies the use of serial EEG recordings perhaps over a period of weeks rather than a single recording.

Although not immediately relevant to the management of children with febrile seizures, the high rate of the various EEG abnormalities mentioned earlier (including the different forms of seizure activity) on long term serial electroencephalographic follow up of children with febrile seizures, means that there is a serious risk of misdiagnosing epilepsy on the basis of such EEG findings alone. Seizure discharge found incidentally in older children should not necessarily be construed as evidence of current epilepsy. Especially in the presence of a history of febrile seizures, such abnormalities may well be simply evidence of a persistent genetically determined electroencephalographic trait unassociated with the risk of afebrile seizures.

\section{Research}

Another possible although very different place for electroencephalographic investigations in children with febrile seizures concerns research, especially the investigation of genetic factors. The morphological similarity mentioned earlier between some of the focal spike or spike wave discharges seen in some children years after the febrile seizures and the centrotemporal spike discharges in benign rolandic epilepsy of childhood has led to the suggestion that there may be some genetic connection between febrile seizures and this form of epilepsy. ${ }^{17}$ Similarly Doose et al have suggested that various genetically determined EEG patterns can be found in a high proportion of patients with febrile seizures, that is, biparietal theta rhythms, spike wave activity in the resting state and on overbreathing, and that provoked by photosensitivity as described earlier. ${ }^{19}$ Alvarez et al suggested that hypnagogic paroxysmal spike wave is also a genetically transmitted EEG pattern. ${ }^{22}$

Genetic studies are not the only type of research needed in the field of febrile seizures. The possibility of defining clinically important subgroups was mentioned earlier in connection with severe myoclonic epilepsy of infants.

Whatever aspect of febrile seizures is researched, as Alvarez and his colleagues rightly point out, ${ }^{22}$ published studies contain clear inconsistencies not only in the criteria used to compare patient groups in different studies of febrile seizures, but in the way electroencephalographic information has been obtained. They are right to recommend, certainly for research purposes, that recordings should be concerned with natural sleep and wakefulness, drowsiness, at least stages 1 and 2 of non-rapid eye movement sleep, arousal, and wakefulness. Failure to standardise the procedure makes comparisons between different studies very difficult. Certainly if this sequence is not followed there will be a failure to demonstrate not only hypnagogic paroxysmal spike wave but other spike wave discharges during sleep with which hypnagogic paroxysmal spike wave might be confused.

Further exploration of these possibilites dependent on electroencephalographic studies would be interesting and justified within the confines of a specific research protocol. Outside the circumscribed clinical and research settings just mentioned electroencephalographic investigations of children with febrile seizures cannot be recommended. 
1 Aicardi J. The medical management of neonatal and infantile seizures and of febrile seizures. In: Porter RJ, Morselli PL, 2 Anonymous. The management of febrile convulsions. Drug Ther Bull 1987;25:9-11.

3 Kendig EL, Dyken PR, Hernandez N, et al. Consensus statement on febrile seizures. In: Nelson KB, Ellenberg JH, eds. Febrile seizures. New York: Raven Press, 1981: 301-6.

4 Menkes JH. Textbook of child neurology. Philadelphia: Lea and Febiger, 1985.

5 Brett EM. Paediatric neurology. Edinburgh: Churchill Livingstone, 1983.

6 Livingstone S. Infantile febrile convulsions. Dev Med Child Newrol 1968;10:374-6.

7 Chevrie JJ, Aicardi J. Duration and lateralisation of febrile convulsions. Etiological factors. Epilepsia 1975;16:781-9.

8 Ellenberg JH, Nelson KB. The efficiency of published recommendations for the treatment of febrile seizures. In: Nelson KB, Ellenberg JH, eds. Febrile seizures. New York: Raven Press, 1981:97-102.

9 Aicardi J, Chevrie JJ. Consequences of status epilepticus in infants and children. In: Delgado-Escueta $A V$, Wasterlain CG, Treiman DM, Porter RJ, eds. Advances in neurology. Vol 34. Status epilepticus. New York: Raven Press, 1983: 115-25.

10 Dravet C, Roger J, Bureau $M$. Severe myoclonic epilepsy of infants. In: Roger J, Dravet C, Bureau M, Dreifuss FE, Wolf $\mathrm{P}$, eds. Epileptic syndromes in infancy, childhood and adolescence. London: Libbey, 1985:58-67.

11 Lennox-Buchtal MA. Febrile convulsions. A reappraisal. Electroencephalogr Clin Neurophysiol 1973;32 (suppl): 1-32.

2 Frantzen E, Lennox-Buchtal MA, Nygaard A. Longitudinal EEG and clinical study of children with febrile convulsions. Electroencephalogr Clin Neurophysiol 1968;24:197-212.

13 Pampiglione G. Prodromal phase of measles: some neurophysiological studies. BMf 1964;ii:1296-300.
14 Wallace SJ, Zealley H. Neurological, electroencephalographic and virological findin

15 Minchom PE, Wallace S. Febrile convulsions: electroencephalographic changes related to rectal temperature. Arch Dis Child 1984;59:371-3.

16 Rantala $H$, Uhari $M$, Tuokko $H$. Viral infections and recurrences of febrile convulsions. $\mathcal{F}$ Pediatr 1990;116: 95-159.

17 Kajitani T, Ueoka K, Nakamura M, Kumanomidou $Y$. Febrile convulsions and rolandic discharges. Brain Dev 1981;3:351-9.

18 Eeg-Olofsson $O$. The development of the electroencephalogram in normal children from the age of 1 through 15 years: paroxysmal activity. Neuropaediatrie 1971;2

19 Doose H, Ritter K, Volzke E. EEG longitudinal studies in febrile convulsions. Neuropediatrics 1983;14:81-7.

20 Tsuboi T, Endo S. Febrile convulsions followed by nonfebrile convulsions. A clinical, electroencephalographic and follow up study. Neuropediatrics 1977;8:209-33.

21 Heijbel J, Blom S, Bergfors PG. Benign epilepsy of children with centrotemporal EEG foci. A study of incidence in with centrotemporal EEG foci. A study
outpatient care. Epilepsia 1975;16:657-64.

22 Alvarez N, Lombroso CT, Medina C, Cantlon B. Paroxysmal spike and wave activity in drowsiness in young children: its relationship to febrile convulsions. Electroencephalogr Clin Neurophysiol 1983;56:406-13.

23 Nelson KB, Ellenberg JH. Prognosis in children with febrile seizures. Pediatrics 1978;61:720-7.

24 Annegers JF, Hauser WA, Elveback LR, Kurland LT. The risk of epilepsy following febrile convulsions. Neurology risk of epilepsy

25 Nelson KB, Ellenberg JH, eds. Febrile seizures. New York: Raven Press, 1981:297-300. 